\title{
A New Training for Older Adults Using Combined Neuromuscular Electrical Stimulation and Volitional Contraction: A Pilot Study
}

\author{
YOSHIO TAKANO, HIROO MATSUSE*, YUUYA TSUKADA*, MASAYUKI OMOTO*, \\ RYUKI HASHIDA* AND NAOTO SHIBA*
}

\author{
Department of Physical Therapy, School of Health Sciences at Fukuoka, International University \\ of Health and Welfare, Okawa 831-8501, *Division of Rehabilitation, \\ Kurume University Hospital, Kurume 830-0011, Japan
}

Received 21 August 2015, accepted 8 February 2016

J-STAGE advance publication 25 May 2016

\begin{abstract}
Summary: The hybrid training system (HTS) resists the motion of a volitionally contracting agonist muscle using force generated by its electrically stimulated antagonist. We have developed a new training method using the principle of HTS. This study was designed to evaluate the effect of HTS with electrical stimulation on muscle strength and physical function by comparing it against training without electrical stimulation in older adults. 16 subjects were randomly divided into two groups: the squat and single leg lift training (control, CTR) group, and the CTR with HTS training group. Some electrical stimulation was applied to the quadriceps and hamstring muscles in the HTS group. The subjects performed training for 25 min per session 3 times a week for 12 weeks. At points before and after the research maximal isokinetic torque, knee-flexors (KFT) and knee-extensors (KET), a one-leg standing test (OLT), a functional reach test (FRT), a 10-meter maximal gait time (10MGT) and Timed up \& go test (TUG) were conducted. None of the subjects had any injuries during the study period. TUG significantly improved after the training period in both the HTS group ( $7.15 \mathrm{sec}$ to $6.01 \mathrm{sec} \mathrm{P}=0.01$ ) and in the CTR.
\end{abstract}

Key words older adults, locomotive training, muscle strength, electrical stimulation, hybrid training system

\section{INTRODUCTION}

The percentage of older people in Japan has been increasing due to the declining birth rate. The National Livelihood Survey 2012 by the Ministry of Health, Labour and Welfare [1] reported that people aged 65 years or older had reached $24.1 \%$ of the population in Japan, and the country is aging more rapidly than any other country in the world. Moreover, according to the Comprehensive Survey of Living Conditions in 2011 of the Ministry of Health, Labour and Welfare, joint disease is the greatest cause of required long-term assistance at $20.7 \%$, and with an added $14.6 \%$ occurrence of fall/fracture, accounting for $1 / 3$ or more of all disabilities of locomotive organs [2]. Therefore, long- term assistance due to locomotor disabilities is one of our biggest problems, and it is clear that prevention of musculoskeletal disease is vital. In 2007, the Japanese Orthopedic Association (JOA) proposed the term "locomotive syndrome (LS)" to designate the condition in which people have a high likelihood of requiring nursing care as a result of disabilities of locomotive organs [3]. Yoshimura et al. [4] conducted a cohort study of 3,040 residents at three locations in Japan in evaluating their risks of the following three disorders: osteoarthritis of the knee, lumbar spinal stenosis and osteoporosis. They estimated that 47 million people aged 40 years and older in Japan have abnormalities associated with one or more of these three disorders detectable with radiography. These 47 million people

Correspondence: Yoshio Takano: Department of Physical Therapy, School of Health Sciences at Fukuoka, International University of Health and Welfare. 137-1 Ebokizu, Okawa Fukuoka 831-8501, Japan. Tel: +81-944-89-2000 Fax: +81-944-89-2001 E-mail: y-takano@iuhw.ac.jp

Abbreviations: ADL, activities of daily living; CTR, control; EMC, electrical muscle contraction; FRT, functional reach test; HTS, hybrid training system; JOA, Japanese Orthopedic Association; KET, maximal isokinetic knee extension torque; KFT, maximal isokinetic knee flexion torque; LS, locomotive syndrome; LT, locomotive training NMES, neuromuscular electrical stimulation; OLT, one-leg standing test; TUG, Timed up \& go test; VMC, volitional muscle contraction; WT, weight training; 10MGT, 10-meter maximum gait speed. 
can be considered to be at risk of LS. LS is often caused by muscle strength decrease due to pain from osteoarthritis of the knee and lack of training caused mainly by age-related weakening of locomotive organs. Thus, a declining change of musculoskeletal system tissues in older people leads to a decline in walking ability and restriction of activities of daily living (ADL), and increases the risk of the need for long-term care. Therefore an effective method of prevention of LS is necessary. Patients with LS require a prevention program that includes physical training, and two kinds of locomotive training (LT); the squat and one-leg standing training are recommended by the JOA, and these are easily performed at home. However, to date few published studies have shown that LT improved physical function in older adults.

Neuromuscular electrical stimulation (NMES) is widely used to improve muscle weakness caused by injury or knee surgery in medicine [5-7]. NMES can also increase muscle strength and hypertrophy in healthy subjects [10]. Recently, the combined application of electrical muscle contraction (EMC) and volitional muscle contractions (VMC) is thought to be more effective than EMC or VMC alone in increasing muscle strength $[8,9]$. The hybrid training system (HTS) resists the motion of a volitionally contracting agonist muscle with force generated by its electrically stimulated antagonist. HTS was developed as a technique to combine the application of EMC and VMC [10-12]. Iwasaki et al. [12] studied the efficacy of HTS compared with conventional weight training (WT) with 15 repetition maximum loads for increasing muscle strength around the knee joint (HTS +25-28\%, WT $+24-33 \%$ ). In older adults, HTS has been shown to produce improvements in muscle strength by about $40 \%$ and mass by about $10 \%$ which is as good as or better than those achieved with a knee flexion machine at $40 \%$ of the maximum VMC [13]. One of the major advantages of HTS is that electrical stimulation can be combined with voluntary activity simultaneously. Therefore we developed a new training method in which HTS could be performed for the squat and single leg lift training (e.g., LT). The purpose of the present pilot study was to examine the effects of HTS during the squat and single leg lift training on muscle strength and physical function in community-dwelling older adults.

\section{SUBJECTS AND METHODS}

\section{Subjects}

The subjects were recruited using posters displayed in local community centers in Okawa City, Fukuoka, Japan. The subjects were given oral and written explanations of the study, including the objectives of the training method and its risks, and all subjects signed consent forms for participation in this research. They were assured that they could give up if they wished. A total of 16 subjects applied. The exclusion criteria for the training intervention were cases of acute orthopedic problems including the knee joint, cerebrovascular or heart disease within the past year, and dementia. The subjects underwent medical and musculoskeletal examinations conducted by a physician. The 16 subjects (6 males and 10 females) with an average age of $71.8 \pm 3.9$ (66-77 years) were randomly divided into two groups by a blinded assessor using a computer: the HTS group and the control (CTR) group. The HTS group consisted of 8 subjects (1 males and 7 females) with an average age of 72.4 \pm 3.1 (ranging 67-71 years), while the CTR group consisted of 8 subjects (5 males and 3 females) with an average age of 71.3 \pm 4.8 (ranging 66-77 years). The Ethics Committee of Kurume University approved the clinical design of this study protocol.

\section{Training protocol}

The training was conducted for 25 minutes per session, 3 times a week for 12 weeks (a total of 36 sessions). Each session was separated by an interval of at least 48 hours. All the training was conducted at the University Laboratory. The subjects were instructed to avoid excessive training during the research period and to carry on their ordinary daily living. During the training, an assistant was always present to provide guidance and monitoring to ensure that the training could be performed safely and properly. The subjects performed the two types of training. In the HTS group, the training consisted of squats (10 minutes) and single leg lifts using HTS (15 minutes). Each training session consisted of 10 sets of 10 reciprocal exercises in both groups. During the squat both hamstrings were stimulated in response to knee flexion, and quadriceps were stimulated in response to knee extension of both legs (Fig. 1). Conversely, during the single leg lift exercise each quadriceps was stimulated in response to hip extension from hip flexion, and each hamstring was stimulated in response to hip flexion (Fig. 2). If the subjects were tired and could not continue training, they were instructed to take breaks at will. The CTR group performed the same training as HTS but without electrical stimulation. 


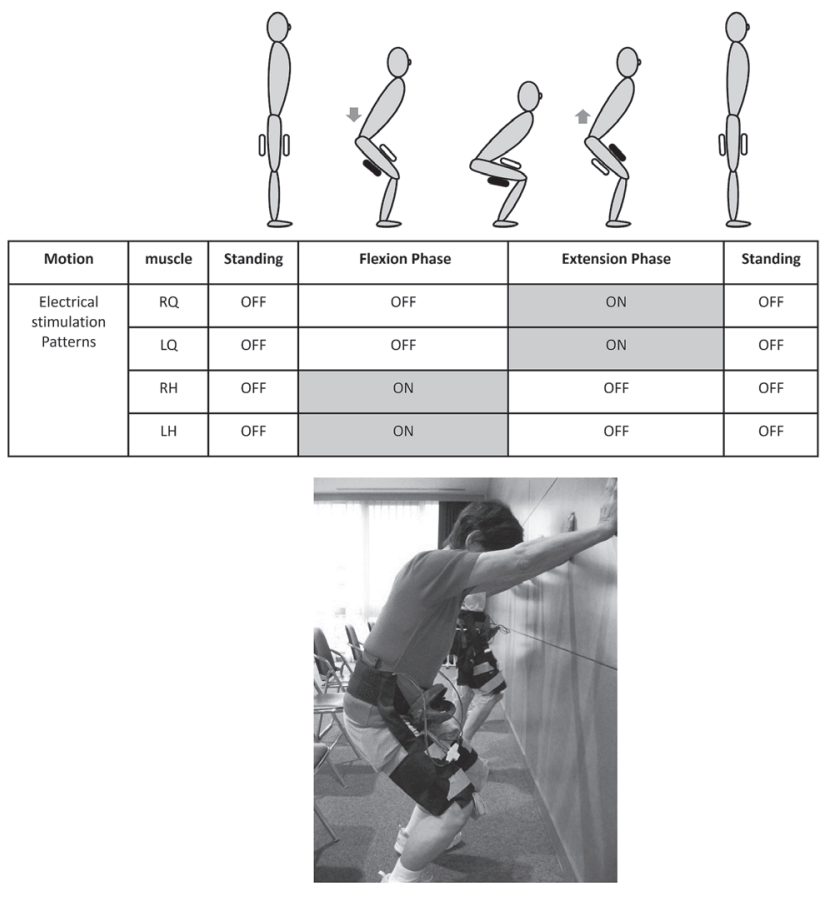

Fig. 1. Electrical stimulation pattern of HTS; the squat training.

The schematic model shows the squat with HTS. During the squat both quadriceps were stimulated in response to knee flexion, hamstrings were stimulated in response to knee extension of both legs.

RQ. Right quadriceps; LQ, Left quadriceps; RH, Right hamstrings; LH, Left hamstrings.

Surface electrodes on thigh.

, Electrodes electrically stimulated;

, Electrodes no electrically stimulated.

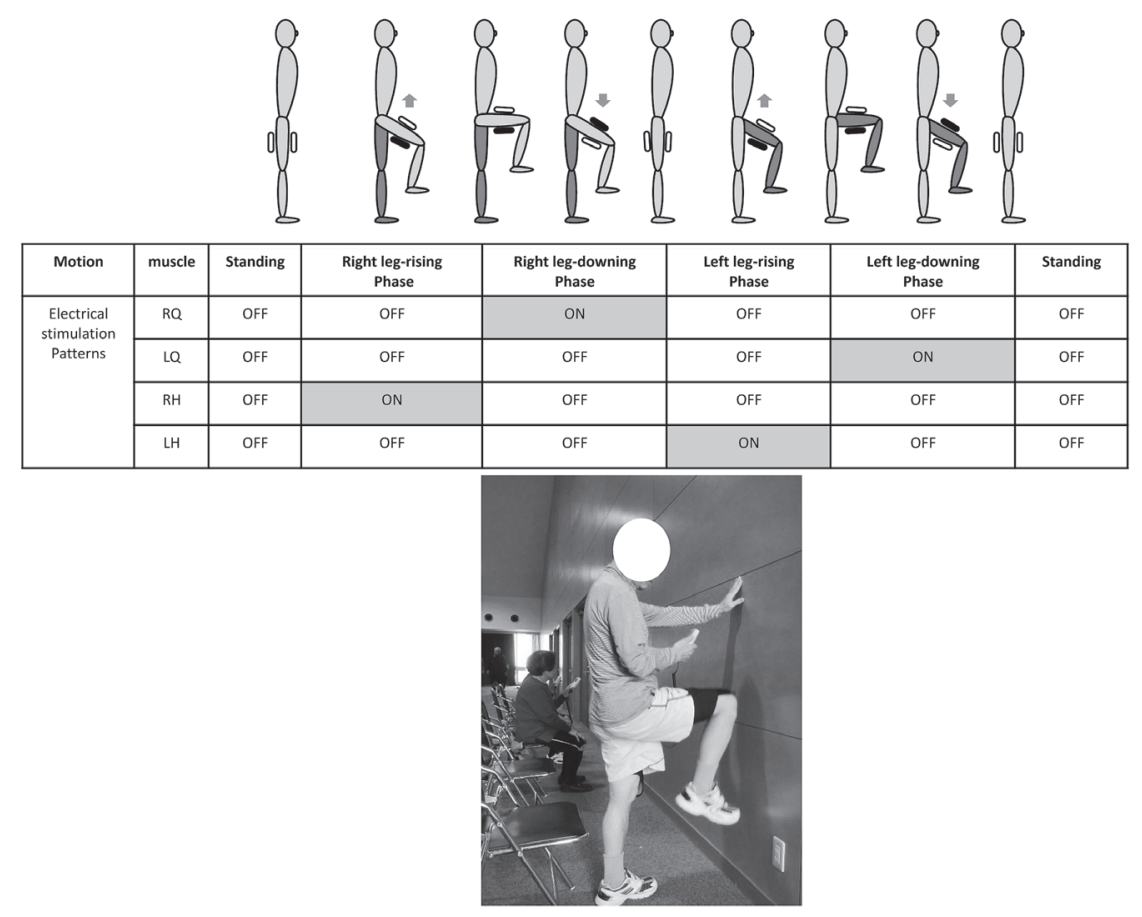

Fig. 2. Electrical stimulation pattern of HTS; the one-leg raising training.

The schematic model shows the one-leg raising with HTS. During the one-leg raising both quadriceps were stimulated in response to hip extension from hip flexion, hamstrings were in response to hip flexion from standing alternately.

RQ. Right quadriceps; LQ, Left quadriceps; RH, Right hamstrings; LH, Left hamstrings.

, Electrodes electrically stimulated;

, Electrodes no electrically stimulated. 


\section{Electrical stimulation protocol}

The target muscles in this study were the quadriceps and hamstring muscles; each leg was stimulated through 2 channels: 4 channels in both legs.

Electrical stimulation device: The electrical stimulation device which has been described previously $[11,12]$ was remodeled for this study by Panasonic Corporation (Home Appliances Development Center Corporate Engineering Division, Appliances Company Panasonic Corporation 2-3-1-2 Noji-higashi, Kusatsu City, Shiga, Japan) (Fig. 3). The device consists of a custom designed waveform generator capable of delivering stimulating signals with unique frequencies and waveforms to as many as 4 pairs of electrodes. Acceleration sensors were used as joint motion sensors (EWTS9PD, Home Appliances Development Center Corporate Engineering Division, Appliances Company Panasonic Corporation 2-3-1-2 Noji-higashi, Kusatsu City, Shiga, Japan). They were placed on the

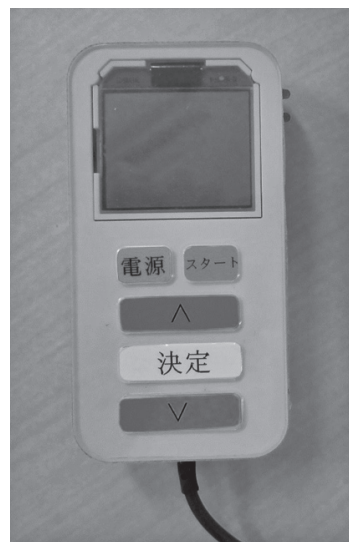

Fig. 3. Electrical stimulation device.

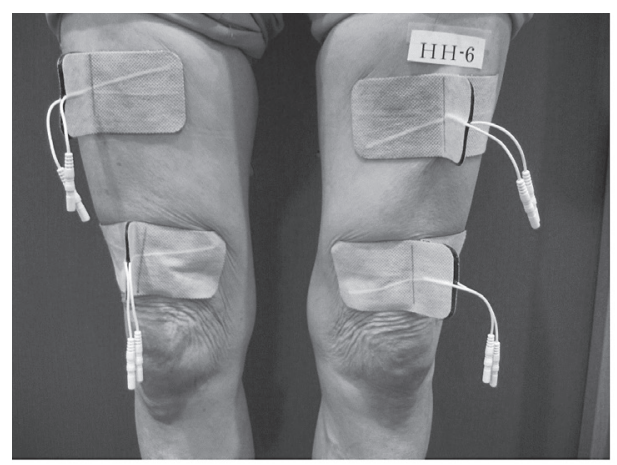

(A) front of each leg $88 \mathrm{~mm}$ above the patellar edge. The device analyzed the algorithm of each motion pattern, and stimulated the antagonist of the motion of each bilateral knee joint during training. Pairs of $5 \times 12-\mathrm{cm}$ low impedance gel-coated silver fiber electrodes (Nihon Medix Co, 315-1, Mukai-machi, Minami-hanashima, Matsudo City, Chiba, Japan.) were placed to widely cover each motor point of the quadriceps and hamstrings (Fig. 4). They were built-in to a quick-drying training suit which the subjects could put on easily.

\section{Electrical Stimulation Parameters}

The stimulation waveform used in this study consists of a $5,000 \mathrm{~Hz}$ carrier frequency with a pulse width of $200 \mu$ s modulated at $40 \mathrm{~Hz}$ ( $2.4 \mathrm{~ms}$ on, $22.6 \mathrm{~ms}$ off) to deliver a rectangular biphasic pulse [14]. The electrical stimulator gives constant voltage stimulus to the human body (regulated voltage). It has a stimulus pattern with interlock and a limiter for safety. Therefore, the effective current is interlocked at $23 \mathrm{mArms}$ when using $500 \Omega$ of the human body equivalent circuit, and the peak voltage and current is limited to under $80 \mathrm{~V}$. Stimulation intensities were re-determined every two weeks during the training period. The training intensities were adjusted to $80 \%$ of the maximum intensity that successfully improved muscle strength and mass without causing pain or numbness [13]. At these electrical stimulation intensities, all subjects were able to walk for 30 minutes.

\section{Evaluations}

All the evaluations were performed with a blind assessor one week before and after the training respectively.

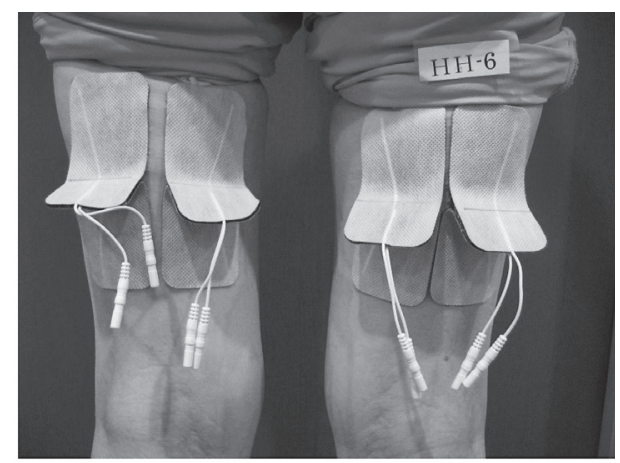

(B)

Fig. 4. Surface electrodes. Electrodes place over motors point each muscle.

A; Knee extensor muscles.

B; Knee flexor muscles. 


\section{Maximal isokinetic torque of knee extension/flexion measurement (KET/KFT)}

All the evaluations were performed by one physical therapist and three assistants. Maximal volitional isokinetic knee extension/flexion torques (KET/KFT) were measured at angular velocities of $60^{\circ} / \mathrm{sec}$ with the Biodex System3-PRO (Biodex Medical Systems Inc., Shirely, NY, USA). During the strength measurements, the subject was seated on the Biodex in an upright position. Velcro belts were applied to fixate the trunk and thigh. The seat was adjusted to the same position at each evaluation. Each session began by establishing that the subject could move his/her lower extremities comfortably throughout the full $10-100^{\circ}$ arc of the training range. They then performed three practice contractions in the direction and at the speed to be tested. A measurement session consisted of 3 sets separated by 3-minutes rest after the practice; the three measurements from the non-dominant lower extremities were pooled, and the mean adjusted by the individual's body weight $(\mathrm{kg})$ was used for statistical analysis.

\section{One-leg standing test (OLT)}

The one-leg standing time was conducted to evaluate balance function [15]. The length of time the subjects were able to stand on their non-dominant lower limb with their eyes open, without any assistance was measured to assess postural steadiness in a static position [16]. Two evaluators performed the measurements, and the measured values provided by the two evaluators were averaged for analysis.

\section{Functional reach test (FRT)}

A functional reach test was conducted to evaluate balance function [17]. The subjects stood straight with one arm stretched out in front at $90^{\circ}$ of shoulder flexion with wrists and fingers straight and palms facing down. The starting position was measured at the tip of the middle finger. The subjects were instructed to reach their hand as far forward as possible without taking a step, and the position of the tip of the middle finger at the end of the reach was recorded. The distance between the starting point and the end point was automatically measured in centimeters with their dominant hand after each practice. The subjects performed the test twice, and the best score was analyzed.

\section{0-meter maximal gait time (10MGT)}

For the evaluation of the 10-meter maximal gait time, 2 meters were added to allow for acceleration be- fore and deceleration after the 10-meter gait test, respectively. The maximal time for the 10-meter gait was measured to evaluate gait speed. Gait speed is a functional assessment tool to show individual activity of daily living or physical capacity $[18,19]$. The subjects were instructed to walk as fast as possible. Measurements were taken by two evaluators, and the times provided by the two evaluators were averaged for analysis.

\section{Timed up \& go test (TUG)}

The timed up \& go test was conducted to evaluate functional mobility [20]. The time it took to rise from a standard chair ( $46 \mathrm{~cm}$ seat height), walk a distance of $3 \mathrm{~m}$, walk back to the chair and sit down were measured. The evaluation was performed twice, and the best score was analyzed.

\section{Statistical Analysis}

All variables are presented as means and standard deviation. Values for KET/KFT, OLT, FRT, 10MGT and TUG were assessed using a Wilcoxon signed rank test in order to compare the differences between pretraining and post-training. All the statistical analyses were performed using JMP Version 9.0 statistical software (SAS Institute Inc., Cary, NC, USA), and p values $<0.05$ were considered to be statistically significant.

\section{RESULTS}

Table 1 shows characteristics of the both groups. All the subjects completed their training sessions without any troubles. During their training sessions, no subjects complained of discomfort or pain. All the results from the parameters are listed in Table 2. KET did not significantly change in either group (HTS group; from $0.99 \mathrm{Nm} / \mathrm{kg}$ pre-training to $1.46 \mathrm{Nm} / \mathrm{kg}$ posttraining, $\mathrm{P}=0.06, \mathrm{CTR}$ group; $1.63 \mathrm{Nm} / \mathrm{kg}$ pre-training to $1.61 \mathrm{Nm} / \mathrm{kg}$ post- training, $\mathrm{P}=0.77$ ). KFT did not significantly change in either group (HTS group; from $0.27 \mathrm{Nm} / \mathrm{kg}$ pre-training to $0.66 \mathrm{Nm} / \mathrm{kg}$ post- training, $\mathrm{P}=0.22$, CTR group; $0.84 \mathrm{Nm} / \mathrm{kg}$ pre-training to 0.78 $\mathrm{Nm} / \mathrm{kg}$ post- training, $\mathrm{P}=0.77$ ). OLT did not significantly change in either group (HTS group; from 4.65 sec pre-training to $7.11 \mathrm{sec}$ post- training, $\mathrm{P}=0.11$, CTR group; $3.33 \mathrm{sec}$ pre-training to $4.17 \mathrm{Nm} / \mathrm{kg}$ post- training, $\mathrm{P}=0.95)$. FRT did not significantly change in either group (HTS group; from $29 \mathrm{~cm}$ pre-training to $25 \mathrm{~cm}$ post- training, $\mathrm{P}=0.36$, CTR group; $36 \mathrm{~cm}$ pre-training to $33 \mathrm{~cm}$ post- training, $\mathrm{P}=0.11$ ). $10 \mathrm{MGT}$ did not significantly change in either group (HTS group; from 5.52 
TABLE 1 .

Subject Characteristics of HTS group and CTR group

\begin{tabular}{lccc}
\hline \multicolumn{1}{c}{ Characteristics } & HTS group $(\mathrm{n}=8)$ & CTR group $(\mathrm{n}=8)$ & $\mathrm{P}$ \\
\hline Mean age $\pm \mathrm{SD}(\mathrm{yr})$ & $72.4 \pm 3.1$ & $71.3 \pm 4.8$ & 0.63 \\
Mean body weight $\pm \mathrm{SD}(\mathrm{kg})$ & $55.9 \pm 11.8$ & $56.1 \pm 13.5$ & 0.78 \\
Mean height $\pm \mathrm{SD}(\mathrm{cm})$ & $152.1 \pm 6.8$ & $162.4 \pm 11.7$ & 0.06 \\
Sex & $(1 / 7)$ & $(5 / 3)$ & NA \\
(male/female) & & &
\end{tabular}

Abbreviations: HTS, hybrid training system. CTR: Control. NA, not applicable. Independent t-test, experimental HTS group vs CTR group

TABLE 2 .

Physical performance in each group at pre- and post-intervention (median (interquartile range))

\begin{tabular}{|c|c|c|c|c|c|c|c|}
\hline \multirow{2}{*}{ Variables } & \multicolumn{3}{|c|}{ HTS group $(n=8)$} & \multicolumn{3}{|c|}{ CTR group $(n=8)$} & \multirow{2}{*}{$\begin{array}{c}\text { P value } \\
\text { between groups }\end{array}$} \\
\hline & Pre & Post & $\mathrm{P}$ & Pre & Post & $\mathrm{P}$ & \\
\hline $\mathrm{KET}(\mathrm{Nm} / \mathrm{kg})$ & $0.99(0.37-1.4)$ & $1.46(0.35-1.84)$ & 0.06 & $1.63(0.52-1.86)$ & $1.61(1.03-1.94)$ & 0.77 & 0.25 \\
\hline KFT (Nm/kg) & $0.27(0.17-0.69)$ & $0.66(0.18-0.73)$ & 0.22 & $0.84(0.6-1.2)$ & $0.78(0.54-1.21)$ & 0.77 & 0.03 \\
\hline OLT (sec) & $4.65(2.15-7.69)$ & $7.11(1.72-33.3)$ & 0.11 & $3.33(1-19.24)$ & $4.17(2.78-8.91)$ & 0.95 & 0.24 \\
\hline FRT $(\mathrm{cm})$ & $29(27-31)$ & $25(24-32)$ & 0.36 & $36(18-45)$ & $33(20-39.5)$ & 0.11 & 0.18 \\
\hline 10MGT (sec) & $5.52(5.07-6.24)$ & $5.54(4.45-5.97)$ & 0.16 & $4.99(3.95-6.46)$ & $5.33(4.43-6.75)$ & 0.37 & 0.49 \\
\hline TUG (sec) & 7.15 (6.25-9.38) & $6.01(5.01-7.79)$ & 0.01 & $7.07(6.3-8.82)$ & $6.89(5.3-8.23)$ & 0.02 & 0.49 \\
\hline
\end{tabular}

Physical performance at a pre-training baseline to the 12-wk conclusion of training, HTS: hybrid training system, CTR: Control, KET: Maximal volitional isokinetic knee extension torque, KFT: Maximal volitional isokinetic knee flexion torque, OLT: One-leg standing test, FRT: Functional reach test, 10MGT: 10-meter maximal gait time, TUG: Timed up \& go test. P values within each group were calculated by the exact Wilcoxon signed rank test. P values between groups were calculated by the rank based analysis of covariance using the ranks for pre-training value.

sec pre-training to $5.54 \mathrm{sec}$ post- training, $\mathrm{P}=0.16$, CTR group; 4.99 sec pre-training to $5.33 \mathrm{sec}$ posttraining, $\mathrm{P}=0.37$ ). In both groups, TUG significantly decreased from pre-training to the end of training (HTS group; $7.15 \mathrm{sec}$ to $6.01 \mathrm{sec} \mathrm{P}=0.01$, CTR group; $7.07 \mathrm{sec}$ to $6.89 \mathrm{sec}, \mathrm{P}=0.02$ ). After the training, there were significant differences between the two groups only in KFT.

\section{DISCUSSION}

The primary objective was to evaluate the present training system to see whether it would improve muscle strength and physical function in older adults. No subjects withdrew from this training, and they all completed their 3 times-a-week, 12-week training program. Significant improvements were observed in the TUG tests in both groups.

Generally, age-related decrease in muscle strength is greater in the lower limbs than in the upper extremities, and it has a major influence on physical perform- ance such as walking ability, standing-up from a seated position and stair-climbing, and leads to disability affecting ADL [21] and falls [22]. The American Geriatrics Society Guideline [23] reported that muscle weakness is a significant risk factor for falls in older adults. Therefore, maintaining the health of locomotive organs is regarded as one of the most important factors for health promotion in older adults. In critical subjects, it is well known that physical training is important for prevention of impairment of locomotive organs (i.e., osteoarthritis, osteoporosis and spondylosis) in older adults [24]. The JOA recommends single leg lifts and squats as prevention training for LS. However, reports of training for LS are limited because of the novelty of the concept of LS. Rhea et al. [25] reported that training twice a week improved physical performance in older adults. It is commonly known that single leg lifts is effective for enhanced balance. The ability to maintain balance is important for older adults, and contributes to walking stability and fall prevention. Sherrington et al. [26] mentioned in a systematic review that bal- 
ance training is most important for reducing fall-rates. In addition, Sakamoto et al. [27] reported a test in which the training group subjects raised and lowered their left and right legs alternately for 1 minute per session. The subjects underwent 3 sessions a day for 6 months. The training group had a significantly reduced fall rate as opposed to the CTR group who did no physical training. In the present study, the training method combined the application of EMC and VMC alternately to the quadriceps and hamstrings. To summarize, traditional electrical stimulation is for the purpose of assisting muscle contraction, but the present training method is for the purpose of providing resistance for the training load in a muscle. For example, during right extremity leg lifts, the right hamstrings are electrically stimulated, and then changes to consist of eccentricEMC on the right hamstrings and VMC on the right quadriceps. Therefore, this method is considered to produce a co-contraction due to the slow leg raising movement (in about 3-seconds). In addition, it is expected that this training will facilitate the improvement of balance ability by working the right and left lower extremities for 15 minutes. The psoas major muscle necessary for improving walking ability (i.e., enables a larger swing phase and improves gait speed) is known to be subject to disuse atrophy due to aging, which in turn contributes to walking dysfunction and falls. Kuno et al. [28] reported that the training of the psoas major influenced walking ability in older adults. Single leg lifts in LT is a static standing hold, and does not lead to resistance training for the psoas major. In order to improve muscle strength for the psoas major, leg lifts should be performed using an attached weight band on the ankle. However, establishing the appropriate training load for the psoas major is difficult and does not always produce effective training. We developed a new electrical stimulation device that provides a resistance load generated by electrical stimulation in the muscle itself, and may be set to an appropriate training load. Therefore, we consider this training method to be more effective than conventional training using a weight band. As a result, we expect leg lifts using the new training method to increase hip flexor muscle strength. In addition, the squat is a widely used LT method regardless of age. There are some studies indicating that the squat differs from knee flexion-extension training in its typical sitting position since it creates the factor of a closed kinetic chain, which co-contracts the agonist and antagonist muscles of the lower extremities $[29,30]$. However, when patients receive poor training instruction, this may produce pain and dysfunction of the joint. For example, it is possible that pain would occur if flexion and extension phase times of the lower extremities are different. Our new training method can prevent these incorrect training methods because it is set at 3 seconds for each movement phase. Thus, this method makes it possible to improve muscle strength more effectively than traditional squats using one's own weight alone. Although this study did not include a detailed pain test, a questionnaire item regarding knee pain suggests that the knee pain was reduced as a result of knee joint stabilization, caused by the elimination of unbalanced muscle contractions. The TUG is a dynamic balance test measuring the ability to stand and of walk, and is quite reliable [31] associated with ADL [19]. We first hypothesized that the significant TUG improvement had resulted from a KET improvement; however, no significant KET improvement was observed in this study. We therefore propose another hypothesis, that the significant TUG improvement can be attributed to neuromuscular adaptation activity induced by the short-time training (i.e., an increase in the motor unit recruitment capacity and motor unit firing rate). Moreover, no significant changes in KET, KFT, OLT, FRT and 10MGT occurred in the HTS group, because of the short training duration. Future studies are needed to clarify the long-term effects of this method on the muscle strength and physical performance in older adults.

Our new method has some strong advantages. First, this training method is based on the principles of HTS, which utilizes a combination of EMC and voluntary VMC. In addition, this method creates resistance to the motion of a volitionally contracting agonist muscle. Second, this method requires small, portable devices, eliminating the need for large equipment such as the WT (e.g., leg press and leg extension machine).

\section{CONCLUSION}

Our new training method, using HTS, showed potential for improving patients' physical performance. Although the sample size was limited, all the subjects completed all of the tests, and there were no dropouts. Therefore, we conclude that the present method is safe to use. In the future, we plan to further investigate the long-term effects of the same training method on physical performance and ADL in older adults.

\section{ACKNOWLEDGMENTS:}

Financial Disclosures:

The authors disclose a potential conflict of interest due to the fact that this study was supported by a $\$ 45,000$ grant from Panasonic Corporation. However, no Panasonic Corporation staff were involved in carrying out the study or in the analysis of re- 
sults. Our purpose was to develop a new and more effective training method for the treatment of people with knee osteoarthritis.

Funding/Support:

These funds were used primarily for personnel expenses, compensation and the stimulator purchase. They were not used for anything except for the expenses of this study.

Institutional Review

The study was designed in accordance with the ethical standards of the Helsinki Declaration of 1975 and received the approval of the Ethics Committee of Kurume University.

All procedures were fully explained to the participants who gave their written informed consent to participate.

\section{REFERENCES}

1. Cabinet Office, Government of Japan, Outline of the results of Aging Society Survey 2013 edition, http://www8.cao. go.jp/kourei/whitepaper/w-2013/zenbun/s1_1_1_01.html (in Japanese)

2. Ministry of Health, Labour and Welfare, Outline of the results of National livelihood Survey 2013. http://www. mhlw.go.jp/toukei/saikin/hw/k-tyosa/k-tyosa13/index.html (in Japanese)

3. Nakamura K. A "super-aged" society and the "locomotive syndrome". J Orthop Sci. 2008; 13:1-2.

4. Yoshimura N, Muraki S, Oka H, Mabuchi A, En-Yo Y et al. Prevalence of knee osteoarthritis, lumbar spondylosis, and osteoporosis in Japanese men and women: the research on osteoarthritis/osteoporosis against disability study. J Bone Miner Metab. 2009; 27:620-628.

5. Rebai H, Barra V, Laborde A, Bonny JM, Poumarat G et al. Effects of two electrical stimulation frequencies in thigh muscle after knee surgery. Int. J SportsMed. 2002; 23:604609.

6. Stevens JE, Mizner RL, and Snyder-Mackler L. Quadriceps strength and volitional activation before and after total knee arthroplasty for osteoarthritis. J Orthop Res. 2003; 21:775779 .

7. Talbot LA, Gaines JM, Ling SM, and Metter EJ. A homebased protocol of electrical muscle stimulation for quadriceps muscle strength in older adults with osteoarthritis of the knee. J Rheumatol. 2003; 30:1571-1578.

8. Dehail P, Duclos C, and Barat M. Electrical stimulation and muscle strengthening. Ann Readapt Med Phys. 2008; 51:441-451

9. Paillard T. Combined application of neuromuscular electrical stimulation and voluntary muscular contractions. Sports Med. 2008; 38:161-177.

10. Yanagi T, Shiba N, Maeda T, Iwasa K, Umezu Y et al. Agonist contractions against electrically stimulated antagonists. Arch Phys Med Rehabil. 2003; 84:843-848.

11. Matsuse H, Shiba N, Umezu Y, Nago T, Tagawa Y et al. Muscle training by means of combined electrical stimulation and volitional contraction. Aviat. Space Environ Med. 2006; 77:581-585.

12. Iwasaki T, Shiba N, Matsuse H, Nago T, Umezu Y et al. Improvement in Knee Extension Strength through Training by Means of Combined Electrical Stimulation and Voluntary Muscle Contraction. Tohoku J Exp Med. 2006; 209:33-40.
13. Takano Y, Haneda Y, Maeda T, Sakai Y, Matsuse H et al. Increasing muscle strength and mass of thigh in elderly people with the hybrid-training method of electrical stimulation and volitional contraction. Tohoku J Exp Med. 2010; 221:77-85.

14. Ward AR, and Shkuratova N. Russian electrical stimulation the early experiments. Phys Ther. 2002; 82:1019-1030.

15. Michikawa T, Nishiwaki Y, Takebayashi T, and Toyama Y. One-leg standing test for elderly populations. J Orthop Sci. 2009; 14:675-685.

16. Jonsson E, Seiger A and Schfeld, H. One-leg stance in healthy young and elderly adults: a measure of postural steadiness? Clin Biomech. 2004; 19:688-694.

17. Duncan PW, Weiner DK, Chandler J, and Studenski S. Functional reach: a new clinical measure of balance. J Gerontol. 1990; 45:192-197.

18. Abellan van Kan G, Rolland Y, Andrieu S, Bauer J, Beauchet $\mathrm{O}$ et al. Gait speed at usual pace as a predictor of adverse outcomes in community-dwelling older people an International Academy on Nutrition and Aging (IANA) Task Force. J Nutr Health Aging. 2009; 13:881-889.

19. Peters DM, Fritz SL and Krotish DE. Assessing the reliability and validity of a shorter walk test compared with the 10-Meter Walk Test for measurements of gait speed in healthy, older adults. J Geriatr Phys Ther. 2013; 36:24-30.

20. Podsiadlo D, and Richardson S. The timed "Up \& Go": a test of basic functional mobility for frail elderly persons. J Am Geriatr Soc. 1991; 39:142-148.

21. Bean JF, Kiely DK, Herman S, Leveille SG, Mizer K et al. The relationship between leg power and physical performance in mobility-limited older people. J Am Geriatr Soc. 2002; 50:461-467.

22. Hughes VA, Frontera WR, Wood M, Evans WJ, Dallal GE et al. Longitudinal muscle strength changes in older adults: influence of muscle mass, physical activity, and health. J Gerontol A Biol Sci Med Sci. 2001; 56:209-217.

23. American Geriatrics Society, British Geriatrics Society, and American Academy of Orthopaedic Surgeons Panel on Falls Prevention. Guideline for the prevention of falls in older persons. J Am Geriatr Soc. 2001; 49:664-672.

24. Hagen KB, Dagfinrud H, Moe RH, Østerås N, Kjeken I et al. Training therapy for bone and muscle health: an overview of systematic reviews. BMC Med. 2012; 19:10-167.

25. Rhea MR, Alvar BA, Burkett LN, and Ball SD. A metaanalysis to determine the dose response for strength development. Med Sci Sports Exerc. 2003; 35:456-464.

26. Sherrington C, Whitney JC, Lord SR, Herbert RD, Cumming RG et al. Effective exercise for the prevention of falls: a systematic review and meta-analysis. J Am Geriatr Soc. 2008; 56:2234-2243.

27. Sakamoto K, Nakamura T, Hagino H, Endo N, Mori S et al. Committee on Osteoporosis of The Japanese Orthopaedic Association. Effects of unipedal standing balance training on the prevention of falls and hip fracture among clinically definedhigh-risk elderly individuals: a randomized controlled trial. J Orthop Sci. 2006; 11:467-472.

28. Takahashi K, Takahashi HE, Nakadaira H, and Yamamoto M. Different changes of quantity due to aging in the psoas major and quadriceps femoris muscles in women. J Musculoskelet Neuronal Interact. 2006; 6:201-205. 
29. Petrella RJ, and Bartha C. Home based exercise therapy for older patients with knee osteoarthritis: a randomized clinical trial. J Rheumatol. 2000; 27:2215-2221.

30. Baker KR, Nelson ME, Felson DT, Layne JE, Sarno R et al. The efficacy of home based progressive strength training in older adults with knee osteoarthritis: a randomized con- trolled trial. J Rheumatol. 2001; 28:1655-1665.

31. Wennie Huang WN, Perera S, Van Swearingen, and Studenski S. Performance measures predict onset of activity of daily living difficulty in community-dwelling older adults. J Am Geriatr Soc. 2010; 58:844-852. 